\title{
On the representation of aerosol activation and its influence on model-derived estimates of the aerosol indirect effect
}

Daniel Rothenberg et al.

Correspondence to: Daniel Rothenberg (darothen@mit.edu)

The copyright of individual parts of the supplement might differ from the CC BY 4.0 License. 


\section{Overview}

In this supplement we provide additional diagnostics and evaluations of MARC, updating some of those previously provided by [Kim et al., 2008] to document the performance of an earlier version coupled to a different global climate model, the NCAR Community Atmosphere Model version 3 (CAM3). For the analysis presented here, we have performed identical simulations to the arg_comp configuration of the model using present-day emissions described in the companion manuscript; however, we have included in the simulation additional online radiative forcing diagnostics which decompose the contributions from each individual MARC mode and the bulk natural aerosols (dust and sea salt).

We restrict our analyses to the final three years of our simulation and focus our attention on both the mean seasonal cycle under constant emissions and the annual average climatology of key fields.

\section{Climatological Distribution of Aerosol}

\subsection{Aerosol Mass Concentrations}

Annual mean total column loadings of the main anthropogenic aerosol modes simulated by MARC are depicted in Figure S1. Abundances of these modes' particles are much higher in the Northern than the Southern Hemisphere as expected, given their primary emissions sources. Weak poleward advection and short residence times limits these aerosols' abundances over the Southern Ocean, although we note that this region does have local direct and precursor sulfate emission sources via dimethyl sulfide (DMS), as well as regional maxima of sea salt emissions. The sulfate here predominantly form NUC-mode particles which do not have a long enough lifetime to supply significant ACC mass.

The BC and OC fields feature emissions hotspots in South America, sub-Saharan Africa, India, and China. Biomass burning activity, which partially emits directly into the BC mode, following [Wang, 2004], helps explain some of this regional variability. Particularly off the western coasts of South America and Africa we see strong transport of BC and OC mass towards clean, maritime regions. To a lesser extent this occurs in the ACC fields as well, although nucleation scavenging preferentially removes some of this aerosol mass as both $B C$ and $O C$ are assumed to be hydrophobic (although impaction scavenging can still deplete them from the atmosphere).

The two mixed modes show a different regional abundance pattern than their externally mixed pure modes, with local maxima shifted towards the Middle East. This co-locates with a lowamplitude local maxima in ACC burden over this area, which stems from direct emissions in Europe which is transported away from their sources. Locally-higher MBS and MOS burdens are also observed in East Asia where there is also a greater abundance of ACC mass. Away from 

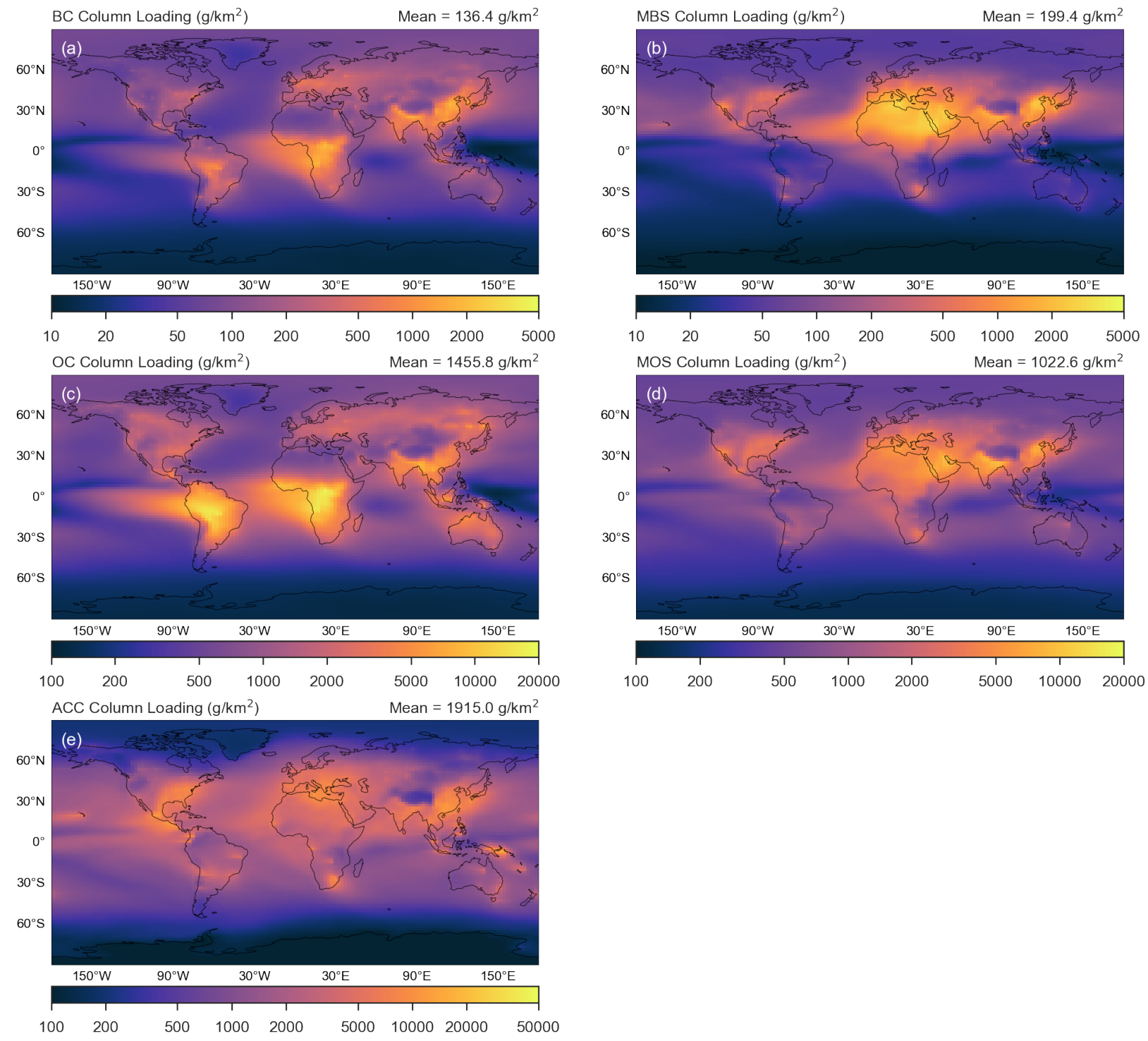

Figure S1 - Annual mean, model-simulated total column mass loadings of (a) external $B C,(b)$ MBS, (c) external OC, (d) MOS, and (e) ACC mode aerosols. All units are in $\mathrm{g} \mathrm{km}^{-2}$. Color schemes are consistent for each row; the total column burdens of NUC and AIT aerosols are small relative to ACC and omitted here.

hotspots near emissions regions, the pure and mixed carbonaceous modes are relatively wellmixed across the Northern Hemisphere, even in the high latitudes.

The annual zonal mean mass burdens of the same MARC modes are shown in height crosssections in Figure S2. Aerosol mass burdens are much greater in the lower troposphere, particularly around latitude bands featuring emissions regions as identified in Figure S1. The carbonaceous and mixed modes feature a sharp latitudinal gradient around $45^{\circ} \mathrm{S}$ throughout the depth of the troposphere, although there is some weaker poleward transport aloft. This is in contrast with the Northern Hemisphere, where poleward of $60^{\circ} \mathrm{N}$ both $\mathrm{OC}$ and $\mathrm{BC}$ modes are 
well-mixed in the lower troposphere, although MBS and MOS mode burdens sharply drop through the extra-tropical storm track region due to locally-higher nucleation scavenging.
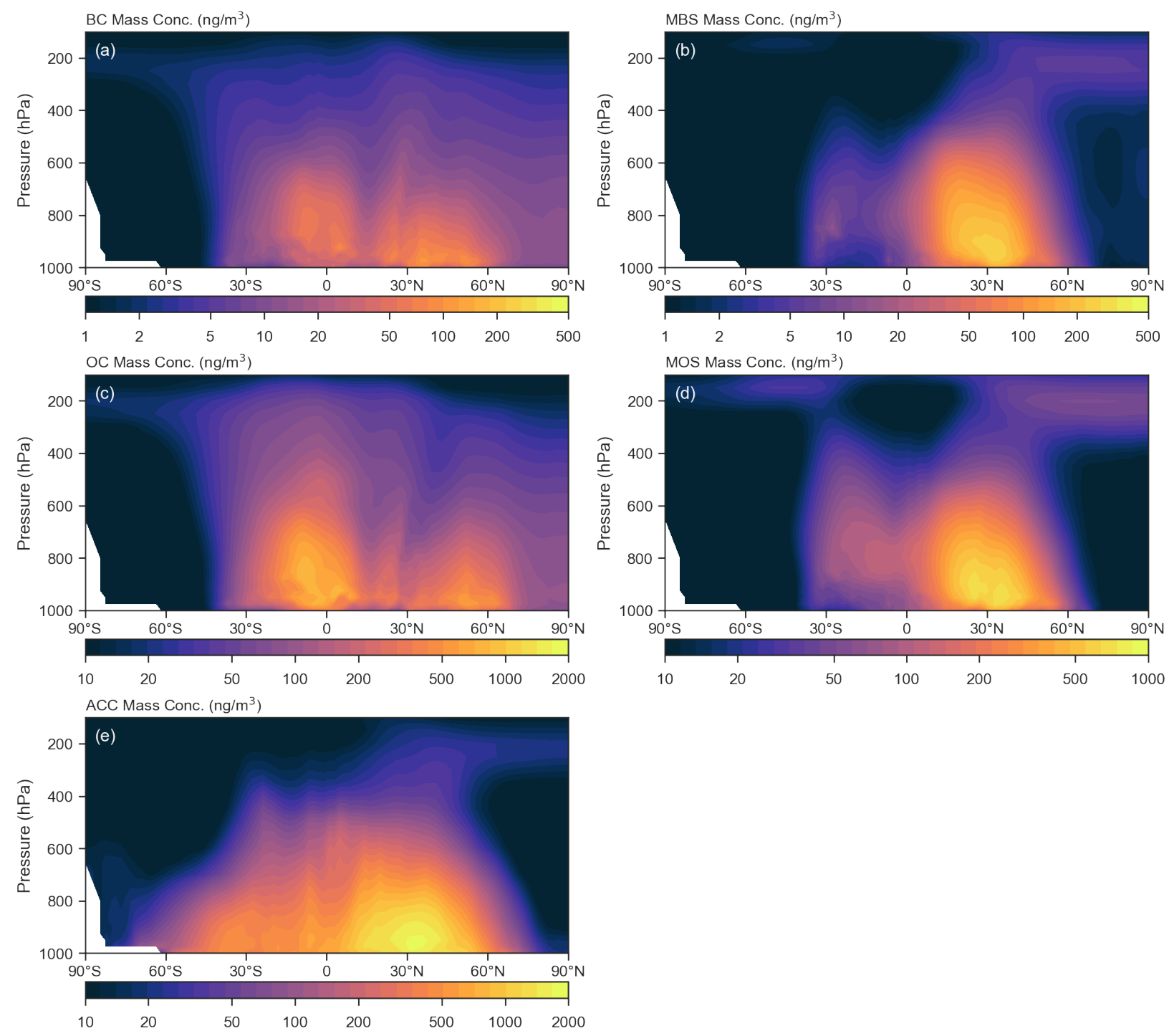

Figure S2 - Annual mean, model-simulated zonal mean mass concentrations of (a) external BC, (b) MBS, (c) external OC, (d) MOS, and (e) ACC mode aerosols. All units are in $\mathrm{ng} \mathrm{m}^{-3}$. Color schemes are different for each plot.

Near the surface, MBS and MOS mass burdens drop sharply across the equator, but some interhemispheric transport does seem to take place above the boundary layer. The secondary maxima in the tropical Southern Hemisphere free troposphere in MOS and MBS further indicates strong local production sources, particularly mixing of available ambient carbon and sulfate particles. However, ACC is more widely distributed in the lower troposphere, and is much more prevalent in the Southern Hemisphere mid-latitudes than the carbonaceous modes. This is in part due to local, natural production of sulfate particles which can grow to ACC sizes. 
Not shown here, the distribution of AIT mode mass maximizes in this region of the SouthernHemisphere, and aloft in the free-troposphere through the tropics - although the in-cell AIT mass burden in these locations is still 2-3 orders of magnitude less than the in-cell ACC mass burden.

Both the MBS and MOS modes as well as the ACC mode are present in the upper-troposphere in the Northern Hemisphere high-latitudes, and the mixed particles to a lesser extent in the same region of the Southern Hemisphere. However, there is a sharp cut-off in the tropics in the meridional distribution of aerosol mass at these altitudes.

\subsection{Aerosol Optical Depth}

Annual mean aerosol optical depth is shown in Figure S3Error! Reference source not found. . Although the global average magnitude of AOD for each of dust, sea salt, and the anthropogenic modes is similar, locally their magnitudes vary dramatically. Over arid regions near the equator, dust AOD is an order of magnitude higher than other species'.

The spatial distribution of AOD further illustrates the geographic extent of the natural versus anthropogenic aerosols; dust is restricted mainly to the African continent with some export to the equatorial Atlantic, whereas sea salt is abundant across the globe - particularly so in the Southern Ocean and downstream of the western boundary currents tracking Asia and North America, in the mid-latitude storm tracks regions. Sea salt abundance is much lower in the tropics and near the inter-tropical convergence zone, where wet scavenging is enhanced and reduces the lifetime of emitted sea salt particles. However, within the Indian Ocean - especially towards the Bay of Bengal - and near the Oceanic continent, sea salt AOD (and abundance) is relatively low.

The distribution of MARC mode AOD is strongly dominated by organic carbon (both external and mixed) and sulfate. An exception to this pattern is in sub-Saharan Africa and South America, where biomass burning contributes greatly to the externally-mixed OC burden and leads to hotspots in optical depth. Sulfate dominates the AOD burden elsewhere around the globe, particularly in Europe and the United States. 

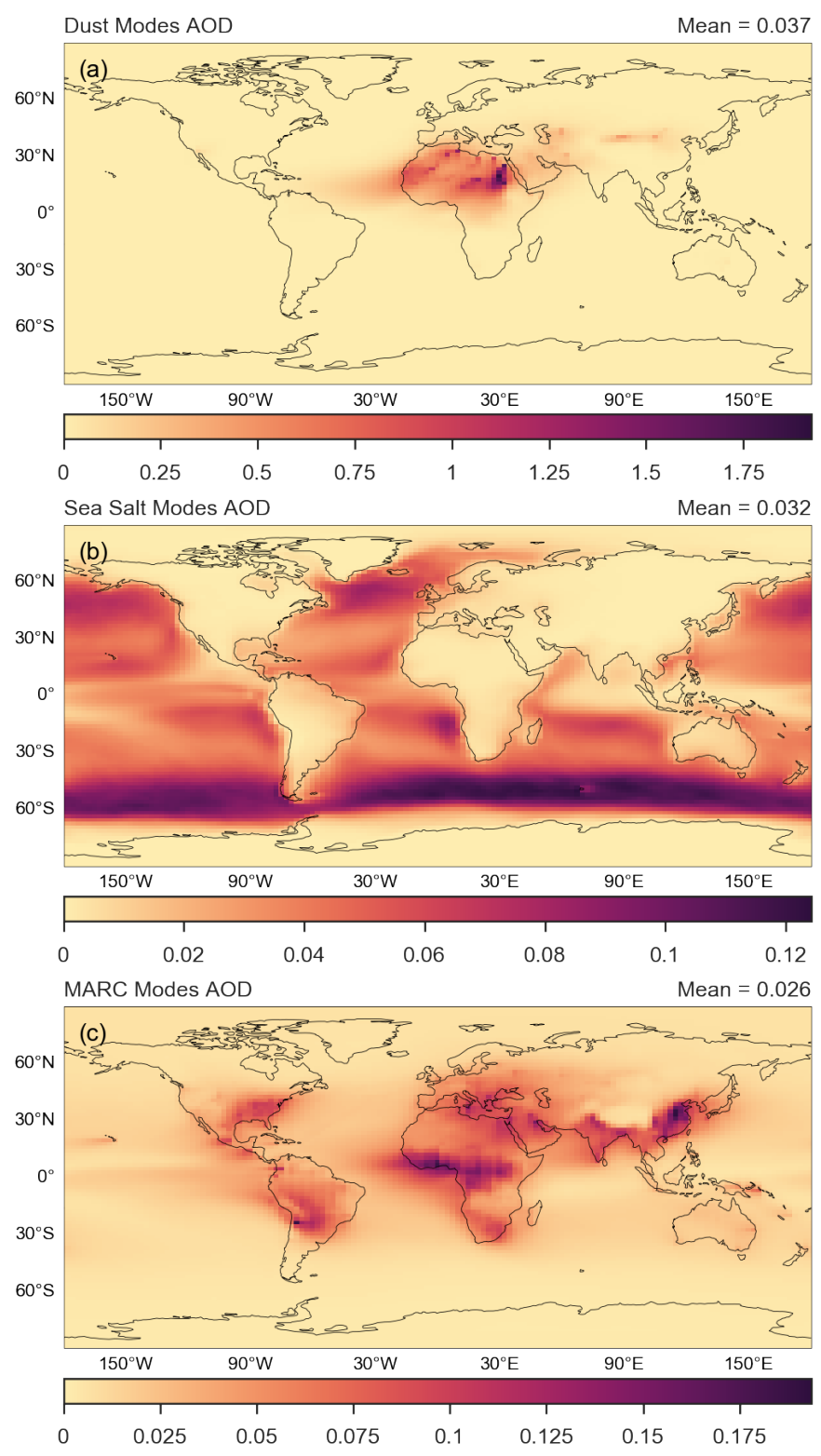

Figure S3 - Aerosol optical depth for (a) bulk dust modes, (b) bulk sea salt modes, and (c) multimoment MARC modes. Note the different colorbar and scaling on each of the plots. All values are unit-less.

\section{Comparison with Observations}

\subsection{Surface Aerosol Mass Concentrations}

We compare model-simulated seasonal-mean surface aerosol constituent mass concentrations at different observing sites across the United States (EPA IMPROVE and CASTNET) and Europe 
(EMEP) in Figures S4Error! Reference source not found.-S6Error! Reference source not found. . In these comparisons, we have taken into account sulfate and both black and organic carbon mass partitioned in the mixed carbon-sulfate modes. That is, for constituent mass concentrations $[X]$ :

$$
\begin{aligned}
{\left[B C_{\text {total }}\right] } & =\left[B C_{\text {external }}\right]+\left[M B S_{B C}\right] \\
{\left[O C_{\text {total }}\right] } & =\left[O C_{\text {external }}\right]+\left[M O S_{O C}\right] \\
{\left[S_{4, \text { total }}\right] } & =[N U C]+[A I T]+[A C C] \\
& +\left(\left[M B S_{\text {total }}\right]-\left[M B S_{B C}\right]\right) \\
& +\left(\left[M O S_{\text {total }}\right]-\left[M O S_{O C}\right]\right)
\end{aligned}
$$

where we have taken advantage of the auxiliary MARC tracer indicating the total mass of organic/black carbon present in each of the mixed carbon-sulfate modes. For each observation site in the three networks, we first compute a monthly climatology for the years between 19952006 where data is available. Similarly, we compute a monthly climatology for each grid cell using the final three years of our MARC simulation. Using these monthly climatologies we further compute seasonal climatologies. We then find the grid cell containing each observation site in each network, and compare the two monthly climatological values.

Overall, sulfate mass simulated by MARC agrees very well with the data taken from all three observation networks. Relative to the CASTNET data, there is a tendency to systematically under-predict sulfate during MAM, but over-predict it during SON and DJF. During DJF, when sulfate is lower across many of the IMPROVE observation sites, there is much more variance in that simulated by MARC, which tends to predict too-high sulfate mass burdens. The agreement among all seasons is better versus the CASTNET data for sulfate, and the model performance over Europe, as evidenced by the EMEP data, is also good.

Within the IMPROVE network, there is a large number of available OC and BC observations. Generally speaking, MARC fares well in comparison with these datasets and there are no systematic biases immediately apparent. Relative to the CASTNET dataset, there is a very weak correlation between MARC-simulated and observed BC; MARC simulates the correct order of magnitude, but displays a seasonal cycle in North American OC which is not present in the observations. This issue is not as readily apparent when compared against the EMEP data; however, MARC tends to systematically under-predict OC mass near the EMEP observation sites. 

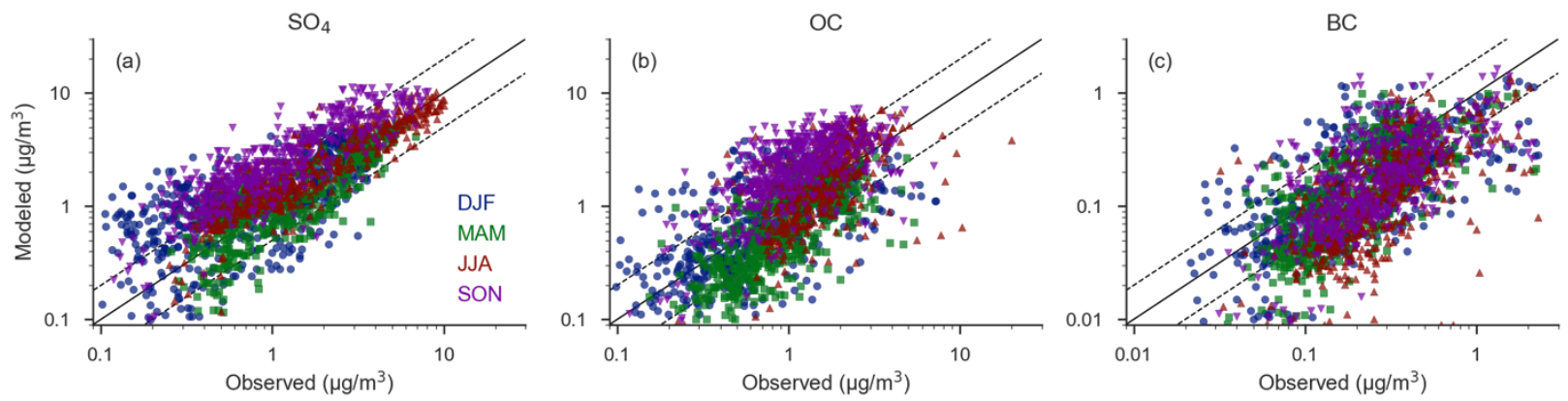

Figure S4 - Comparison between observed and model-simulated seasonal-mean surface (a) sulfate, (b) organic carbon, and (c) black carbon mass concentrations. The solid and dashed black lines represent the one-to-one line and factors of 2 and 0.5. Glyph coloring denotes season. Observations taken from EPA IMPROVE network.
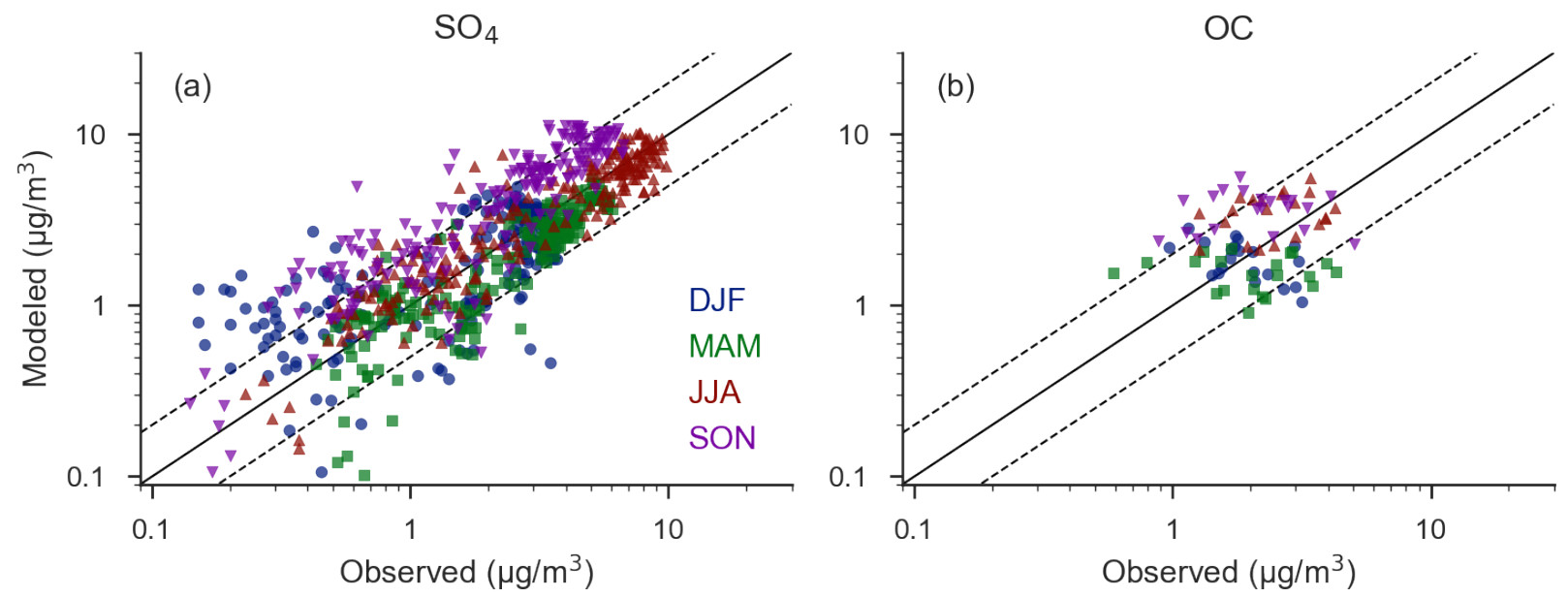

Figure S5 - Similar to Figure S4 Error! Reference source not found. but using data from the EPA CASTNET network, using available monthly mean.
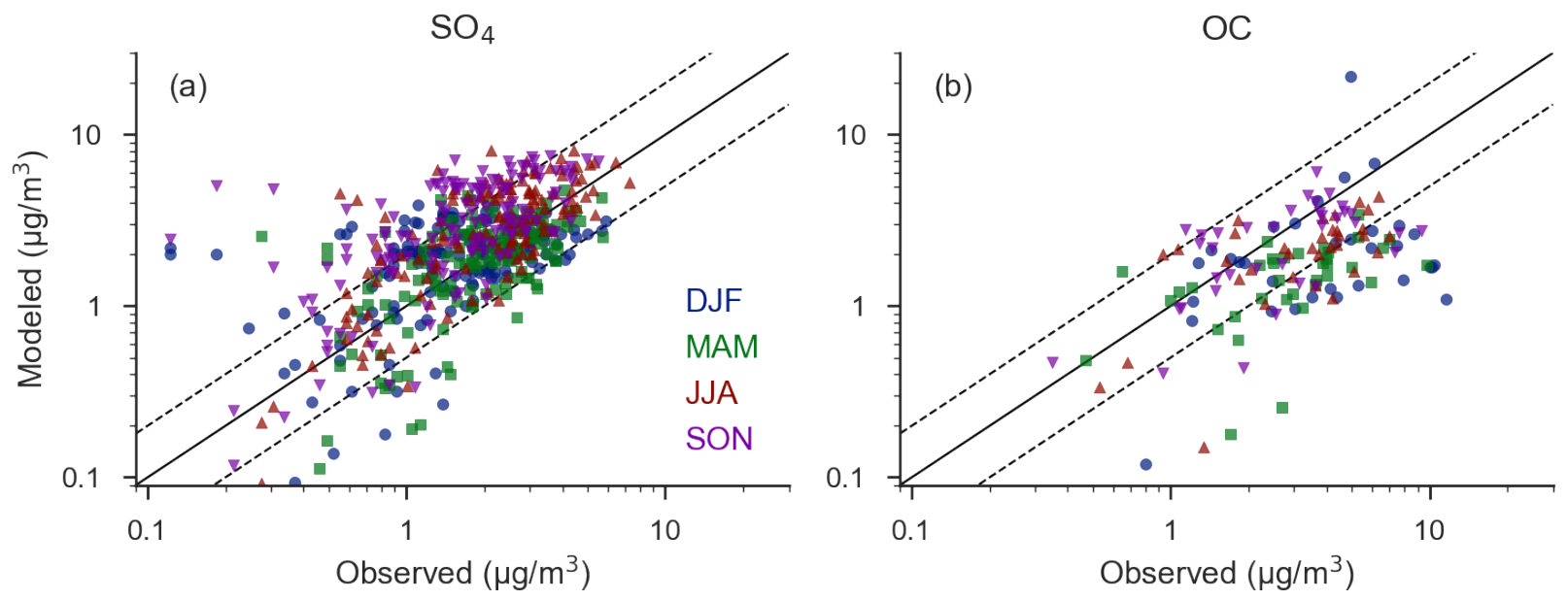

Figure S6 - Similar toError! Reference source not found. Figure S4 but using data from the EMEP network, using available monthly mean data. 
Summary statistics for the relationships depicted here can be found in Table S1. The model much more capably simulates the seasonality of sulfate mass burden in North America than in Europe, given the lower $r^{2}$ values when compared against the EMEP data in all seasons. Model error statistics are much worse for OC (with near- $0 r^{2}$ for EMEP in DJF and CASTNET in MAM); this suggests there may be some systematic biases in the OC simulation by the model. However, because of the limited definition of OC in MARC, there may also be a bias in terms of comparing different species in substances in the observational data versus model output.

BC data was only available from the IMPROVE network. Similar to the case with OC, the correlation between seasonal BC for MARC and IMPROVE is relatively low in all seasons. The $r^{2}$ statistic, however, is likely influenced by the very large variance in the relationship between the two datasets.

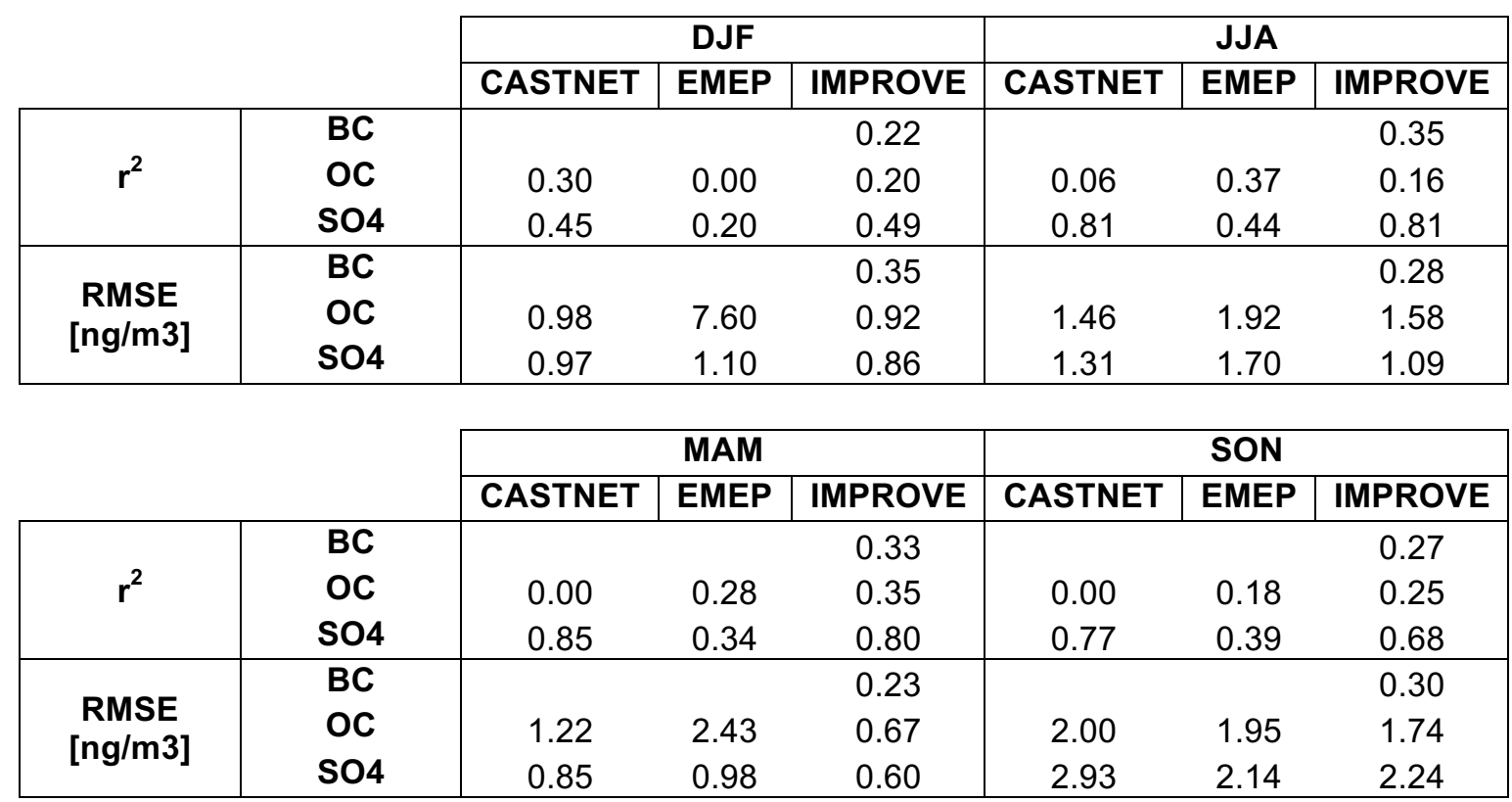

Table S1 - Summary statistics for seasonal-mean surface observations simulated by MARC versus reference observation networks highlighted in Figures S4Error! Reference source not found.-S6Error! Reference source not found.

\subsection{Vertical Distribution of Black Carbon}

To assess MARC's simulation of the vertical distribution of black carbon, we compare against mass concentrations analyzed from data collected during the High-performance Instrumented Airborne Platform for Environmental Research (HIAPER) Pole-to-Pole (HIPPO) campaign [Schwarz et al., 2010]. For each flight, we binned the observations by altitude in $500 \mathrm{~m}$ segments, and then further grouped into five zonal bands. To compare with MARC, we first interpolated the monthly mean $\mathrm{BC}$ mass output fields produced by MARC onto a local altitude 
coordinate. We then performed the same binning/grouping operation. We only considered MARC grid cells within a range that contains the bulk of the zonal extent of the globe sampled during the HIPPO campaign, between $150^{\circ} \mathrm{E}-170^{\circ} \mathrm{E}$. The resulting profiles are plotted in Figure S7Error! Reference source not found..

MARC generally over-predicts BC, especially in the free troposphere. However, it does a relatively good job capturing the magnitude of surface $B C$ concentrations across latitude bands and seasons and the overall vertical distribution of $\mathrm{BC}$. We note that in many flights, the distribution of observed $\mathrm{BC}$ has an extremely heavy positive tail with a few relatively large data points; this explains why in many cases, the mean HIPPO curve is close to the $75^{\text {th }}$ percentile or outside of $25^{\text {th }}-75^{\text {th }}$ percentile range altogether.

The largest $B C$ over-predictions tend to occur in the Southern Hemisphere and in the upper troposphere/lower stratosphere regions. A possible explanation for this discrepancy could be that the rate of wet scavenging - especially in deep convection - could be too small in the model. Towards the poles in both hemispheres the model does a much better job at capturing the distribution of BC. This is particularly the case in the Arctic, where outside of the late Summer months (HIPPO V) the model very well reproduces the observed distribution of BC throughout the lower and middle troposphere. 

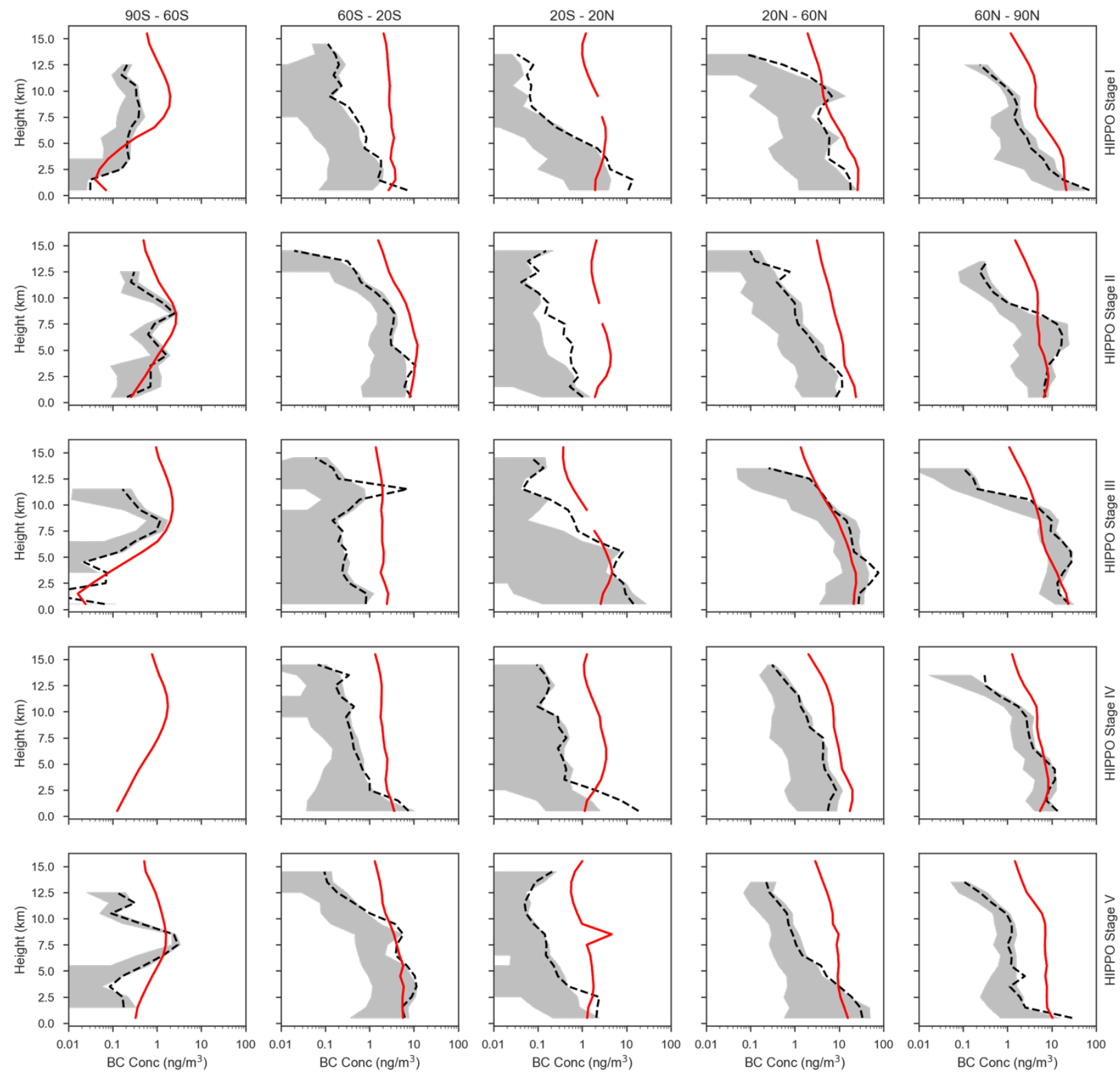

Figure S7 - Averaged vertical profiles of BC mass concentration for different latitudinal bands, compared with profiles averaged from HIPPO observations. Each HIPPO stage is plotted in a separate row: Stage I (January), Stage II (October - November), Stage III (March-April), Stage IV (June - July), and Stage V (August - September). Red curve indicates average MARC profile; dashed black curve indicates mean HIPPO profile; gray shaded area indicates the 25-75 percentile range of all HIPPO observations binned to produce the profile in that plot. 


\subsection{Aerosol Optical Depth}

Model-simulated aerosol optical depth is evaluated against the Aerosol Robotic Network (AERONET, http://aeronet.gsfc.noaa.gov), which is comprised of observations taken from seventy-six sites across the globe, and observations taken aboard the MODIS Terra satellite (processed into monthly climatologies on a 1-degree grid). MARC's default estimates of AOD are taken at a wavelength of 550nm, so we convert the AERONET observations using the available observations taken at 500nm and the measured Ångström exponent,

$$
\tau_{550}=\tau_{500}\left(\frac{550}{500}\right)^{-\alpha}
$$

We include in our estimates of AOD the simulated natural aerosol fields (dust and sea salt) produced by MARC. However, we note that biases in these natural aerosol fields could substantially influence the comparison versus AERONET, particularly because the local dust burden can swamp the signal in AOD produced by anthropogenic aerosols. The results of our comparison with AERONET data can be found in Figure S8Error! Reference source not found..
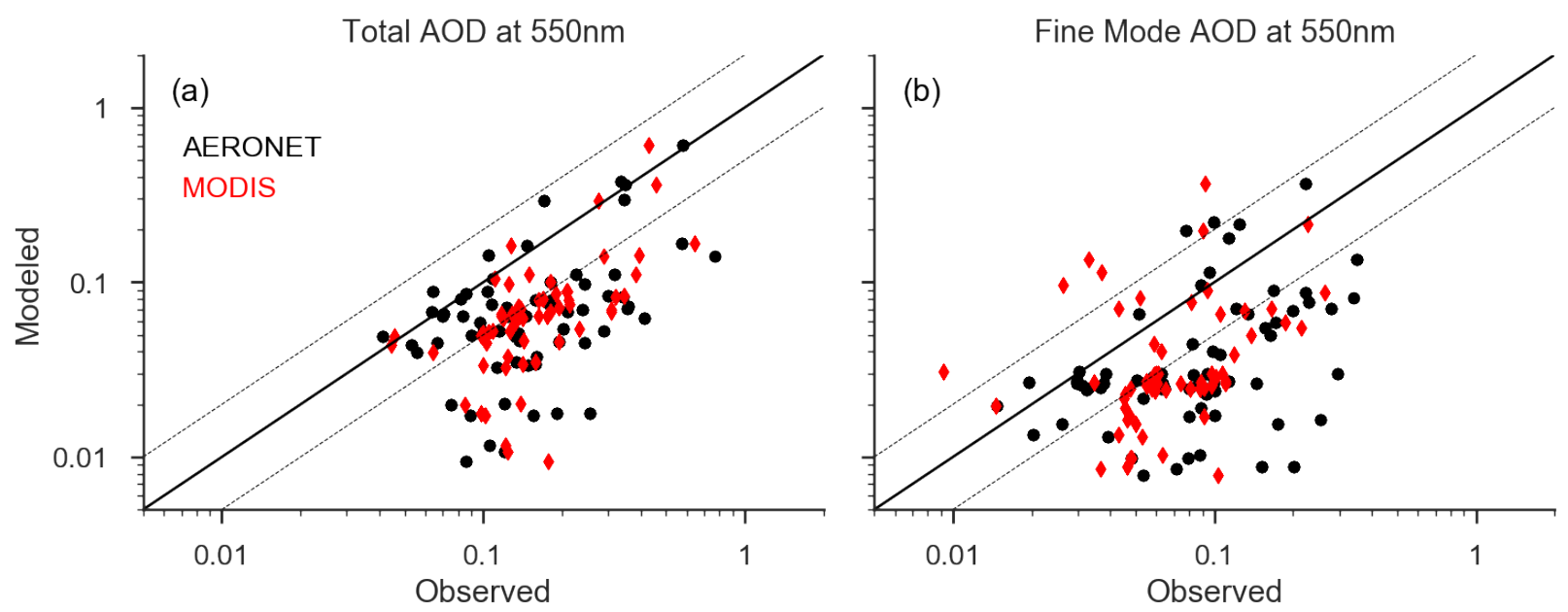

Figure S8 - Modeled and observed annual (a) total and (b) fine-mode fraction mean aerosol optical depth at 550nm. Observations are taken from AERONET and MODIS, as indicated by the colored glyphs. All data are unit-less. Solid line indicates one-to-one correspondence, with dashed lines indicating error factors of 2 and 0.5 , respectively.

Overall, MARC under-estimates AOD at many locations. However, a further breakdown by AERONET region and season shows that this bias is most pronounced in the Winter and Spring, albeit with clusters of sites where MARC simulates AOD much more faithfully than at others. The overall under-prediction statistics are exacerbated by particularly low AOD simulated in South America and near maritime regions. Over the United States, Europe, North Africa, Australia, and Asia, the model much more faithfully reproduces observed AOD values. 
Other factors beyond the climatology of aerosol mass/number distributions could influence the discrepancy between observed and modeled AOD. In particular, some of the regions where the discrepancy is the largest are where $O C$ and MOS particles yield important contributions to total AOD. Within MARC, we assume fixed optical properties for OC and adjust for ambient relative humidity. However, for the MOS particles, we assume a homogenous population of uniformly mixed particles, and compute optical properties based on the fractional mass of sulfate and organic carbon in each particle. These assumptions could play an important role in influencing the integrated optical properties of the aerosol size distribution in regions where $\mathrm{OC} / \mathrm{MOS}$ dominate, and further influence the diagnosed radiative forcing there.

The simulated AOD performs slightly better when compared against the MODIS data. Overall, the correlation coefficient between annual mean AOD derived at AERONET and MODIS sites is 0.41 for total AOD but 0.14 for fine-fraction AOD. For total AOD, the correlation between MARC and MODIS and AERONET is 0.38 and 0.34 , respectively; these correlations decrease to 0.08 and 0.13 for fine-fraction AOD. The performance with MODIS is exacerbated for the fine mode aerosols because the observations show much less variance than those simulated by MARC.

\section{Direct Radiative Forcing}

In Figure S9 we present a decomposition of the annual mean shortwave direct radiative forcing by the major anthropogenic aerosol modes simulated within MARC. These differ from the direct radiative forcing presented in the companion manuscript in that (a) radiative forcing from natural aerosol is neglected here, and (b) the radiative forcing decomposition in the manuscript adopts the methodology of [Ghan, 2013] to account for surface albedo influences and the vertical distribution of aerosol.

Within MARC, the external black carbon mode exerts a modest positive radiative forcing, which is maximized near BC emission sources. Its mixture with sulfate, however, shows strong regional variability and a much smaller global average. In regions where sulfate is prevalent such as the USA, Europe, and Eastern China, the MBS mixture (which assumes a BC core coated with a shell of sulfate) is a net scatterer of shortwave radiation. In other regions where there is more BC such as North Africa and the Arabian Peninsula, the absorbing core of the MBS particles dominates. These contrasting effects nearly balance each other, but produce a net positive radiative forcing.

The remaining aerosol modes are all net coolers and exert a negative direct radiative forcing. The total negative forcing from both the $\mathrm{OC}$ and MOS modes nearly equals the total sulfate negative forcing. That sulfate forcing is wholly dominated by the influence of the ACC mode; the smaller NUC and AIT modes produce an order of magnitude smaller radiative forcing, and those particles (even in the regions aloft where they are relatively more abundant) have only a small influence on the simulation. 
Compared with the earlier version of MARC documented by [Kim et al., 2008], the total direct radiative forcing in the MARC-CESM is slightly smaller in magnitude, as the total positive forcing from $B C$ and $M B S$ is smaller and the large negative forcing from sulfate has been reduced.
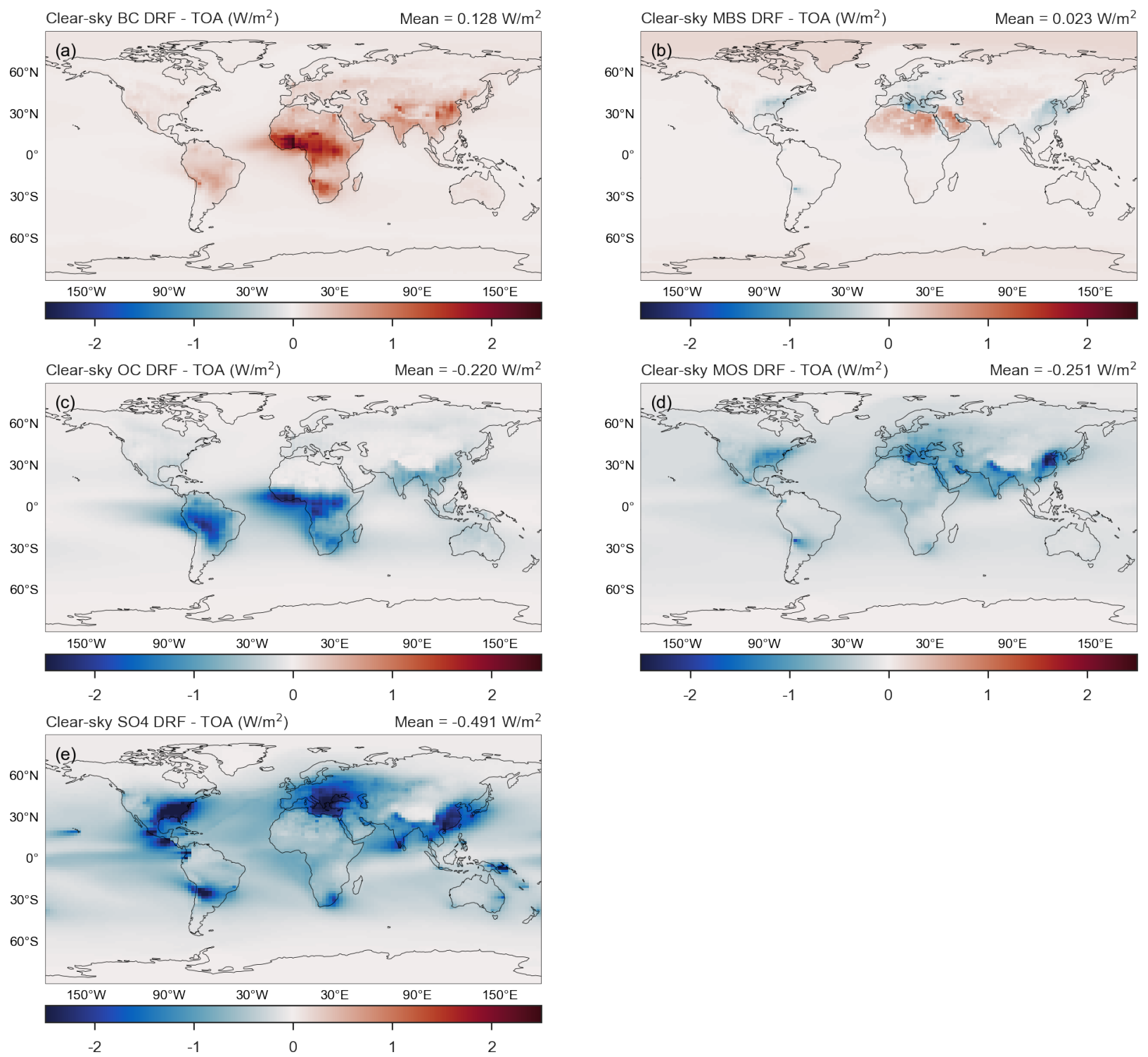

Figure S9 - Annual mean clear-sky shortwave direct radiative forcing of: (a) black carbon, (b) mixed black-carbon sulfate, (c) organic carbon, (d) mixed organic-carbon sulfate, and (e) total sulfate aerosol modes estimated at the top of the atmosphere. The color bar and scale is consistent for all plots. 


\section{References}

Ghan, S. J. (2013), Technical Note: Estimating aerosol effects on cloud radiative forcing, Atmos. Chem. Phys., 13(19), 9971-9974, doi:10.5194/acp-13-9971-2013.

Kim, D., C. Wang, A. M. L. Ekman, M. C. Barth, and P. J. Rasch (2008), Distribution and direct radiative forcing of carbonaceous and sulfate aerosols in an interactive size-resolving aerosol-climate model, J. Geophys. Res., 113(D16), D16309, doi:10.1029/2007JD009756.

Schwarz, J. P., J. R. Spackman, R. S. Gao, L. A. Watts, P. Stier, M. Schulz, S. M. Davis, S. C. Wofsy, and D. W. Fahey (2010), Global-scale black carbon profiles observed in the remote atmosphere and compared to models, Geophys. Res. Lett., 37(18), doi:10.1029/2010GL044372.

Wang, C. (2004), A modeling study on the climate impacts of black carbon aerosols, J. Geophys. Res., 109(D3), D03106, doi:10.1029/2003JD004084. 